\title{
Horizontal gene transfer and genome evolution in Methanosarcina
}

\author{
Sofya K. Garushyants ${ }^{1}$, Marat D. Kazanov ${ }^{1}$ and Mikhail S. Gelfand ${ }^{1,2^{*}}$
}

\begin{abstract}
Background: Genomes of Methanosarcina spp. are among the largest archaeal genomes. One suggested reason for that is massive horizontal gene transfer (HGT) from bacteria. Genes of bacterial origin may be involved in the central metabolism and solute transport, in particular sugar synthesis, sulfur metabolism, phosphate metabolism, DNA repair, transport of small molecules etc. Horizontally transferred (HT) genes are considered to play the key role in the ability of Methanosarcina spp. to inhabit diverse environments. At the moment, genomes of three Methanosarcina spp. have been sequenced, and while these genomes vary in length and number of protein-coding genes, they all have been shown to accumulate HT genes. However, previous estimates had been made when fewer archaeal genomes were known. Moreover, several Methanosarcinaceae genomes from other genera have been sequenced recently. Here, we revise the census of genes of bacterial origin in Methanosarcinaceae.

Results: About $5 \%$ of Methanosarcina genes have been shown to be horizontally transferred from various bacterial groups to the last common ancestor either of Methanosarcinaceae, or Methanosarcina, or later in the evolution. Simulation of the composition of the NCBI protein non-redundant database for different years demonstrates that the estimates of the HGT rate have decreased drastically since 2002, the year of publication of the first Methanosarcina genome.

The phylogenetic distribution of HT gene donors is non-uniform. Most HT genes were transferred from Firmicutes and Proteobacteria, while no HGT events from Actinobacteria to the common ancestor of Methanosarcinaceae were found. About $50 \%$ of HT genes are involved in metabolism. Horizontal transfer of transcription factors is not common, while $46 \%$ of horizontally transferred genes have demonstrated differential expression in a variety of conditions. HGT of complete operons is relatively infrequent and half of HT genes do not belong to operons.

Conclusions: While genes of bacterial origin are still more frequent in Methanosarcinaceae than in other Archaea, most HGT events described earlier as Methanosarcina-specific seem to have occurred before the divergence of Methanosarcinaceae. Genes horizontally transferred from bacteria to archaea neither tend to be transferred with their regulators, nor in long operons.
\end{abstract}

Keywords: Horizontal gene transfer, Archaea, Bacteria, Genome evolution, Methanosarcina

\section{Background}

Horizontal gene transfer (HGT), also known as lateral gene transfer, plays a major role in the evolution of microbial genomes. It helps microorganisms to rapidly acquire new metabolic capabilities and adapt to environmental changes [1-3]. Most genes involved in HGT are associated

\footnotetext{
* Correspondence: gelfand@iitp.ru

'A.A. Kharkevich Institute for Information Transmission Problems, RAS, Bolshoi Karetny per. 19, build.1, Moscow 127051, Russia

${ }^{2}$ Faculty of Bioengineering and Bioinformatics, M.V. Lomonosov Moscow State University, Vorobievy Gory 1-73, Moscow 119991, Russia
}

with pathogenesis, symbiosis, metabolism, and antibiotic resistance [4].

HGT in bacteria is relatively well-studied and several estimates of its rate have been published [5-11], while horizontal transfer of genes from bacteria to archaea is less well characterized, but was shown to be important for origin of major archaeal clades [12].

HGT was shown to occur between both closely and distantly related organisms [13, 14]. According to the complexity hypothesis, horizontal transfer of genes encoding proteins with many protein-protein interactions

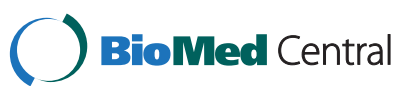


is relatively infrequent as compared to HGT of genes encoding proteins with fewer interactions $[15,16]$. Besides, genes tend to be transferred between genomes with similar codon usage [17].

HGT between bacteria and archaea was shown to be possible almost for all genes, except for a small fraction of genes toxic to the recipient organism [18].

Methanosarcina genomes are among the largest among archaea. It has been suggested that the large genome size in this genus is caused by massive HGT from bacteria [19]. Other members of the Methanosarcinaceae family are a psychrophile Methanococcoides burtonii [20], and halophiles Methanohalobium evestigatum, Methanohalophilus mahii [21], Methanolobus psychrophilus, Methanosalsum zhilinae, and Methanomethylovorans hollandica. All these species except the latter have much shorter genomes than Methanosarcina spp.

Methanosarcinaceae, like all Methanosarcinales, inhabit diverse environments and possess the largest set of metabolic pathways among Archaea. Methanosarcinales share such traits as acetoclastic methanogenesis, the presence of cytochromes, genes encoding the $\mathrm{A}, \mathrm{K}$, and $\mathrm{N}$ subunits of reduced coenzyme F420 (F420H2) dehydrogenase, bacterial-type phosphoglycerate mutase, bacterial adenylate kinase, nonhistone chromosomal protein $\mathrm{MC1}$ involved in chromosome condensation, and the long variant of condensin subunit ScpB [22].

Horizontal transfer of a short operon from Clostridia $[14,23]$ was shown to dramatically change Methanosarcinales [14] or Methanosarcinaceae [23] metabolic capabilities, and allowed these organisms to use methyl compounds as substrates for methanogenesis. Strikingly, the first observations of possible HGT in Methanosarcina mazei showed that almost all functional types of genes could be horizontally transferred from bacteria to Methanosarcina spp. [19, 24, 25] and as much as $30 \%$ of genes in $M$. acetivorans were predicted to be of bacterial origin [19]. About $50 \%$ of genome of $M$. burtonii was shown to have atypical oligonucleotide composition and high transposon content, suggestive of HGT [20].

Analysis of specific patterns of gene gain in Archaea was performed using the arCOG database [26], and by phylogenetic tree reconstruction [27]. In both cases, some groups, and in particular Haloarchaea, Methanomicrobia and smaller taxonomic groups of methanogenic archaea were shown to acquire substantially more genes (more than a thousand for Haloarchaea) than others. While Methanosarcinales had been shown to acquire hundreds of metabolic genes from eubacteria by HGT [26, 27], the origin of such genes and their location in genome, as well as HGT events in the common ancestors of Methanosarcinaceae and Methanosarcina was not studied in detail. Here, we revisit the estimates of the HGT rate in Methanosarcina spp. and Methanosarcinaceae, and characterize possible donors of HT genes, their functions, operon structure, and gene expression.

\section{Results}

\section{HGT identification}

Groups of orthologous proteins (GOPs) were built for all Methanosarcina spp., as well as for all Methanosarcinaceae with similarity cut-offs $50 \%$ and $40 \%$, respectively (Table 1).

All GOPs with more than half proteins annotated as transposases or transposon-associated proteins were removed from the data (30 Methanosarcina GOPs and 38 Methanosarcinaceae GOPs). The final database contained Methanosarcina GOPs that cover about $65 \%$ of Methanosarcina proteins (2778 GOPs); $94 \%$ (2624) of GOPs contained only one orthologous protein per organism; the rest contained paralogs (co-orthologs) for at least one species. From initial Methanosarcinaceae GOPs all GOPs that were already a part of Methanosarcina GOPs were removed, and only GOPs that have at least one protein from Methanosarcina spp., $55 \%$ of the initial Methanosarcinaceae GOPs (1702; 9993 proteins from 6 species), were retained in the database. After that, 1375 Methanosarcinaceae GOPs included in the database did not contain paralogs.

All GOPs containing bacterial-type genes were selected using BLASTP (for details see Materials and Methods). For each selected GOP up to 100 top BLAST hits were aligned. Using this alignments, two sets of phylogenetic trees were constructed, neighbor-joining trees with bootstraps, and maximum likelihood trees, totaling 736 tree sets (Fig. 1a,b). If the Methanosarcina proteins were on the clade that contained Methanosarcina and bacterial proteins but no other archaeal proteins, the corresponding genes were considered to be laterally transferred from bacteria. Finally, we identified 349 Methanosarcina genes from 143 GOPs as likely laterally transferred from bacteria to the Methanosarcina last common ancestor. In addition, genes from 72 GOPs were transferred from bacteria to the last common ancestor of all Methanosarcinaceae. We further analyzed singletons (genes present in only one Methanosarcina spp.) and found that 14 genes were transferred recently in $M$. acetivorans; 33 genes were transferred in M.barkeri, and 10, in M.mazei. In M. barkeri we observed horizontal transfer of an operon comprised of four bacterial-type CRISPR-associated proteins (Additional file 1). Overall, $221 \mathrm{HT}$ genes were found in $M$. acetivorans; $214 \mathrm{HT}$ genes, in M. barkeri; 151, in M. mazei (Table 2 and Additional file 1). Hence, according to our estimates, about $5 \%$ of genes in Methanosarcina were horizontally transferred from bacteria to either the last common ancestor of Methanosarcinaceae or Methanosarcina, or to one of the Methanosarcina species. 
Table 1 Statistics for initial orthologous groups in Methanosarcinaceae

\begin{tabular}{|c|c|c|c|c|c|}
\hline & \multirow[b]{2}{*}{$\begin{array}{l}\text { \# of proteins in } \\
\text { the genome }\end{array}$} & \multicolumn{2}{|c|}{ Methanosarcinaceae GOPs (3122) } & \multicolumn{2}{|c|}{ Methanosarcina GOPs (2808) } \\
\hline & & $\begin{array}{l}\text { \# of proteins in } \\
\text { initial GOPs }\end{array}$ & $\begin{array}{l}\text { \# of orphan } \\
\text { proteins }\end{array}$ & $\begin{array}{l}\text { \# of proteins } \\
\text { in GOPs }\end{array}$ & $\begin{array}{l}\text { \# of orphan } \\
\text { proteins }\end{array}$ \\
\hline Methanococcoides burtonii DSM 6242 & 2273 & 1811 & 462 & - & - \\
\hline Methanohalobium evestigatum Z-7303 & 2254 & 1699 & 555 & - & - \\
\hline Methanohalophilus mahii DSM 5219 & 1987 & 1713 & 274 & - & - \\
\hline Methanosarcina acetivorans C2A & 4540 & 3325 & 1215 & 2852 & 1688 \\
\hline Methanosarcina barkeri str. Fusaro & 3624 & 3062 & 562 & 2548 & 1076 \\
\hline Methanosarcina mazei Go1 & 3370 & 2888 & 482 & 2537 & 833 \\
\hline
\end{tabular}

The number of GOPs in each category of groups is presented in brackets in the table header

The $M$. mazei genome is the smallest one in the genus, and only 82 genes in $M$. mazei remain that have been horizontally transferred to the last common ancestor of Methanosarcina spp. This is almost twice fewer than the number of genes transferred to the other two species (141 in M. acetivorans, 128 in M. barkeri).

The phylogeny of Methanosarcinaceae was reconstructed using 16S RNA [28, 29] and by conserved archaeal proteins [30]. These phylogenetic trees are not completely congruent. The phylogenetic trees based on proteins involved in translation and on 23S rRNA (see Methods, Fig. 2a,b) show that M. mazei and $M$. acetivorans are more closely related to each other than to $M$. barkeri, while the phylogenetic tree based on 16S rRNA clusters together $M$. acetivorans and $M$. barkeri to the exclusion of M. mazei (Fig. 2c). We found 12 GOPs containing HT genes only from $M$. mazei and $M$. acetivorans, and 67 GOPs containing HT genes only from $M$. acetivorans and $M$. barkeri, while no GOPs contained HT genes only from M. mazei and $M$. barkeri. If the $16 \mathrm{~S}$ tree reflects the evolution correctly, then multiple HGT events had occurred in the common ancestor of M.acetivorans and M. barkeri, otherwise if the protein-based and 23S rRNA trees are correct, then $M$. mazei has lost a considerable fraction of genes horizontally transferred to the Methanosarcina last common ancestor.

\section{Time-scaled simulation of the non-redundant DB composition and the predicted HGT rate}

The rate of the observed HGT to the last common ancestor of Methanosarcina or Methanosarcinaceae turned out to be drastically lower than that reported in previous studies $[19,25]$. Two possible explanations for that may be, first, insufficient sensitivity of our procedure or, second, changes in the database composition. To select between these possibilities, HGT searches against a series of time-stamped databases were implemented. The number of bacterial genes in each database was fixed at the 2011 level, while the number of genes from archaea varied, so that, e.g., the 2001 database contained all archaeal genes sequenced strictly before 2002. In order to make the results consistent with the published data, all bacterial-like genes selected after the BLAST search were considered a

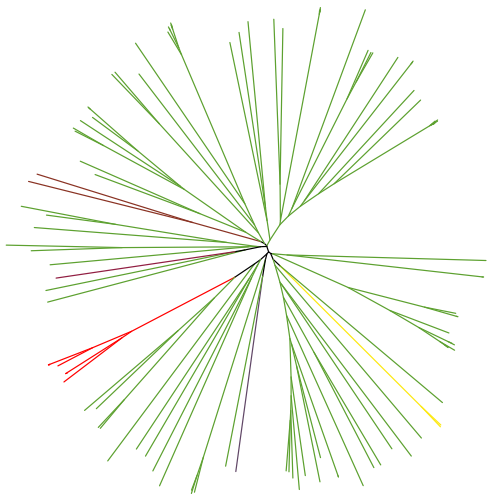

b

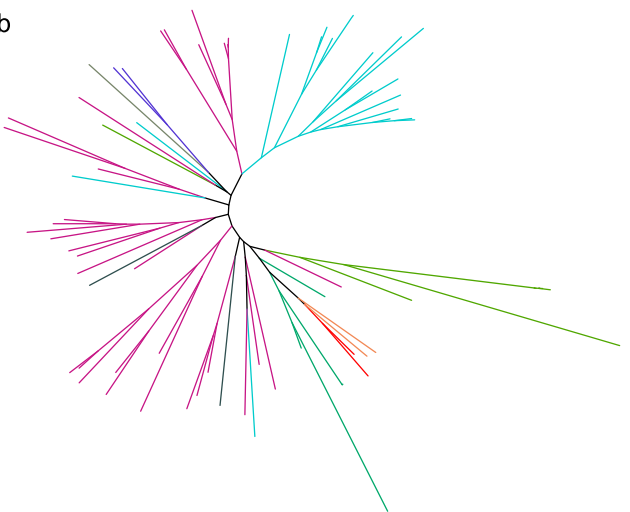

Fig. 1 Phylogenetic trees for some GOPs. Phylogenetic trees constructed by maximum-likelihood algorithm for a acetate kinase AckA, involved in acetoclastic methanogenesis, and $\mathbf{b}$ uncharacterized alpha/beta hydrolase (PF12695). Methanosarcina spp. are shown in red, other Methanosarcinaceae in orange, Firmicutes in light green, Actinobacteria in blue, Synergistetes in brown, Thermotoga in dark-purple, Proteobacteria in purple, Dictyoglomi in yellow, Cyanobacteria in emerald, and Aquificae in grey. Other archaea are shown in dark blue 
Table 2 Summary of predicted HT genes in Methanosarina spp

\begin{tabular}{llllll}
\hline HGT in & $\begin{array}{l}\text { \# of } \\
\text { GOPs }\end{array}$ & $\begin{array}{l}\text { \# of } \\
\text { genes }\end{array}$ & $\begin{array}{l}\text { Genes } \\
\text { in MA }\end{array}$ & $\begin{array}{l}\text { Genes } \\
\text { in MB }\end{array}$ & $\begin{array}{l}\text { Genes } \\
\text { in MM }\end{array}$ \\
\hline $\begin{array}{l}\text { Methanosarcinaceae } \\
\text { Methanosarcina }\end{array}$ & 72 & 178 & 66 & 53 & 59 \\
$\begin{array}{l}\text { HGT in one of the } \\
\text { Methanosarcina species }\end{array}$ & - & 57 & 14 & 33 & 10 \\
All & 215 & 586 & 221 & 214 & 151 \\
\hline MA - M. acetivorans, MB - M. barkeri, MM - M. mazei & &
\end{tabular}

MA - M. acetivorans, MB - M. barkeri, MM - M. mazei

HT, without subsequent tree construction. The estimated fraction of candidate bacterial-origin genes in the last common ancestor of Methanosarcina spp. drastically decreases from $37 \%$ (928 ORFs for M. mazei) as if in 2001 to $8 \%$ in 2011 (222 ORFs for M. mazei). Additionally, $7.5 \%$ of genes in 2011 (194. ORFs) seem to be horizontally transferred in the last common ancestor of Methanosarcinaceae. Figure 3 presents the data on $M$. mazei only, as in previous works the number of HGT events was calculated only for this species. Because in this experiment only genes in GOPs were analyzed, a smaller portion of HT genes in 2001 were obtained, 928 ORFs versus 1043 ORFs in previously published results [19].

The drastic change in the predicted rate of HGT to Methanosarcina is seen in the 2006 time-stamped database, when two Methanosarcinaceae genomes were sequenced, and events that could be earlier assigned as HGT to the last common ancestor of Methanosarcina turned out to be more ancient. Hence, the HGT effect on the Methanosarcina genomes after their divergence from other Methanosarcinales species probably has been overestimated in previous studies, and the HGT rate observed here seems to be more realistic. While the present analysis does not directly address the issue of the total number of genes of bacterial origin in the Methanosarcina genomes, this simulation demonstrates that it has likely been overestimated in early studies.

\section{The first control case: HGT in Thermotogaceae}

Thermotoga maritima was also considered to be highly prone to HGT, as it had been claimed that $24 \%$ of genes in these thermophilic bacterium had been transferred from archaea [31]. Later it was shown that the fraction of possible HGT genes in Thermotoga spp. is 8-11\%, but the BLAST e-value cutoff applied by the authors $\left(10^{-4}\right)$ was of low stringency [32]. We applied our BLAST-based pipeline to revisit these results. GOPs were built for proteins of all sequenced Thermotogaceae: Thermotoga spp., Thermosipho spp., Petrotoga mobilis, Kosmotoga olearia, and Fervidobacterium nodosum. All singletons and GOPs containing transposases were removed. Then for all GOPs that contained $T$. maritima proteins, the time-stamped pipeline was run, but in this case the numbers of both archaeal and bacterial genomes for each year-stamped database were changed. We found only 28 of 1761 analyzed GOPs (less than $1 \%$ of all genes in genome) to be of possible archaeal origin, transferred to the common ancestor either of the genus or the family. This is smaller than the earlier estimates [31-34].

Then we repeated the procedure not for GOPs, but for all T. maritima proteins. When the 1999 database with the lowest cut-offs was considered, only $10 \%$ (188 ORFs) of $T$. maritima genes were seen as possibly horizontally transferred, fewer than 451 in the original publication [31], and for the 2008 database this number is only $4.6 \%$ (86 ORFs), again lower than the previously observed number of 204 ORFs [32].

Our possible explanation for this discrepancy could be that the 1999 study had considered individual genes, then constituting a large fraction of database entries, while we analyzed only complete genomes.

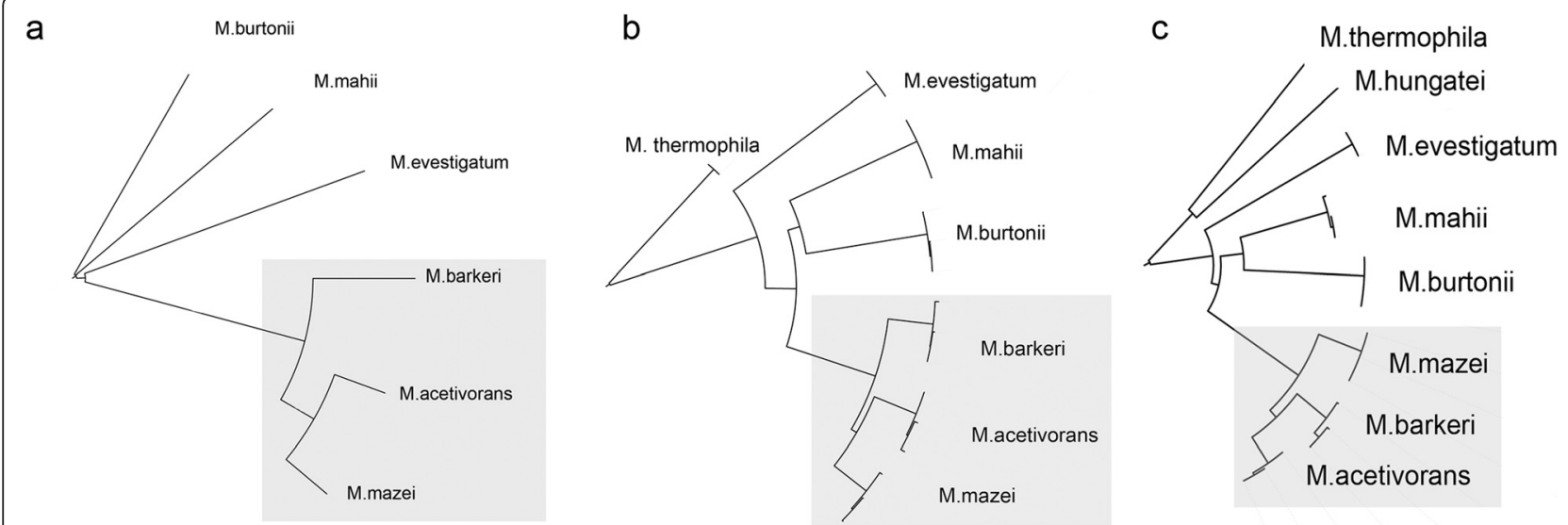

Fig. 2 Phylogenetic trees for Methanosarcinaceae. Phylogenetic trees constructed by neighbor-joining algorithm are based on ribosomal proteins (a), $23 \mathrm{~S} \mathrm{rRNA}(\mathbf{b})$, and $16 \mathrm{~S} \mathrm{rRNA}(\mathbf{c})$ 


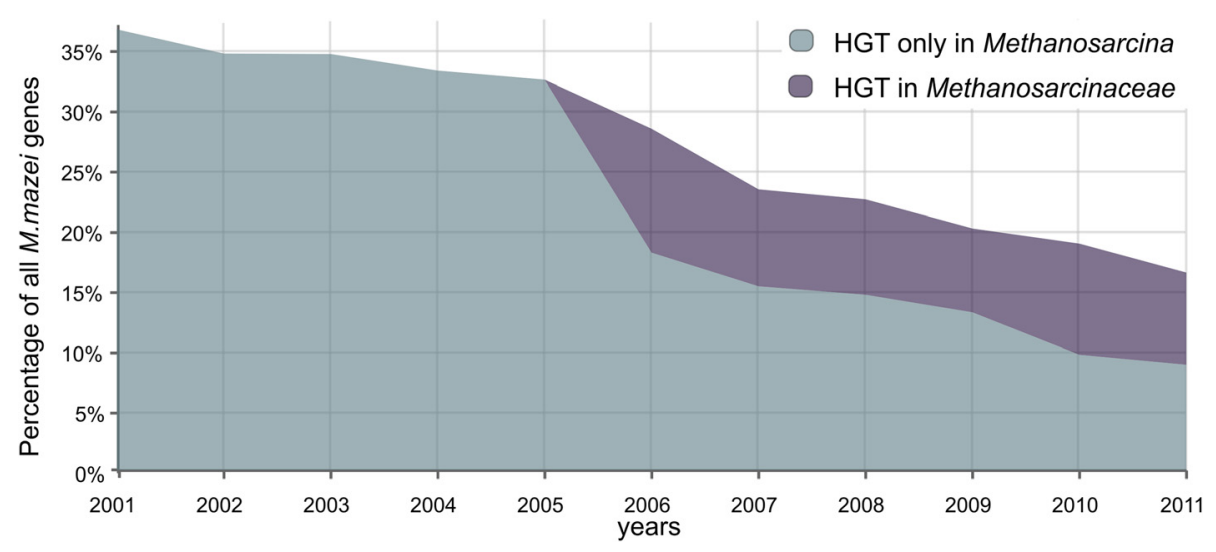

Fig. 3 Relation between the estimated frequency of HGT and the year of DB release. To make the results consistent with the published data [17], HGT predictions were based on the results of BLAST search only, without subsequent tree construction, hence, the shown fractions for 2011 are higher than mentioned in the text. Blue area - HGT in Methanosarcina spp., purple - HGT in Methanosarcinaceae; see the text for definitions

The second control case: HGT in Thermococcaceae

To validate the approach against false positives we ran the HGT prediction procedure on sequenced Pyrococcus genomes. Pyrococcus spp. (family Thermococcaceae) are well-studied, and thought to have small genomes with a low rate of HGT. Indeed, the arCOGs analysis demonstrated massive gene gain in the last common ancestor of Thermococcales, but the origin of these genes was not discussed, although HGT from other archaeal clades was shown to be possible [26]. To identify possible events of HGT from bacteria, we applied the pipeline using the same settings as for the Methanosarcina spp. All Pyrococcus spp. genes were included in GOPs, no singletons were found, and $76 \%$ of Pyrococcus GOPs contained exactly one orthologous protein per genome, while the rest contained paralogs in at least one species. One HGT event to the last common ancestor of Thermococcaceae involving a hypothetical protein was observed. The predicted low HGT rate in Pyrococcus spp. shows that the genomes of Methanosarcinaceae are indeed unusually dynamic.

\section{Taxonomic distribution of transferred genes}

We further attempted to identify possible sources of bacterial genes horizontally transferred to the last common ancestor of Methanosarcina spp. or Methanosarcinaceae. Firmicutes and Proteobacteria were shown to be frequent donors of horizontally transferred genes: 104 GOPs contain genes transferred from Firmicutes with 12 of them further assigned to Clostridia, and 6, to Bacilli; 66 GOPs arose from Proteobacteria; 12, from Planctomycetes; 6, from the Bacteroides/Chlorobi group; 6, from Actinobacteria; 9, from Cyanobacteria etc. (Table 3). For 46 GOPs and 9 singletons, the origin could not be determined exactly. Though Actinobacteria is the third best-sequenced taxon, HGT from this group were rare and occurred only in the last common ancestor of Methanosarcina, moreover, all transferred genes were subsequently lost in $M$. mazei.

As Firmicutes and Proteobacteria have the largest number of sequenced representatives, this can lead to overestimation of their effect. Indeed, it has been shown that the number of unique COGs increases with the addition of new organisms to the pan-genome [35, 36], so well-sequenced taxa have more unique genes in their pan-genomes. To offset that, the overrepresentation coefficient was calculated as the number of GOPs containing genes transferred from a given taxon divided by the

Table 3 Taxonomy distribution of horizontally transferred genes

\begin{tabular}{llll}
\hline Group & In Methanosarcina & In Methanosarcinaceae & All \\
\hline Firmicutes & 79 & 25 & 104 \\
Proteobacteria & 50 & 16 & 66 \\
unclassified bacteria & 27 & 28 & 55 \\
$\begin{array}{l}\text { Planctomycetes; } \\
\text { Planctomycetacia }\end{array}$ & 10 & 2 & 12 \\
$\begin{array}{l}\text { Cyanobacteria } \\
\text { Bacteroidetes/Chlorobi }\end{array}$ & 3 & 2 & 9 \\
group; Bacteroidetes & & 3 & 6 \\
Actinobacteria & 6 & & \\
Synergistetes & 4 & 0 & 6 \\
Acidobacteria & 4 & 0 & 4 \\
Chlamydiae/ & 3 & 0 & 4 \\
Verrucomicrobia group & & 0 & 3 \\
Thermotoga & 1 & 0 & 1 \\
Chloroflexi & 1 & 0 & 1 \\
Deferribacteres & 1 & 0 & 1 \\
Deinococcus-Thermus; & 0 & 0 & 0 \\
Deinococci & & & \\
\hline Methonosarinain this case corrpondstoeither & &
\end{tabular}

Methanosarcina in this case corresponds to either the last common ancestor of Methanosarcina, or even more recent HGT in one of the Methanosarcina species 
number of sequenced genomes for this taxon (Fig. 4). This procedure implicitly assumed linear growth of the pan-genome size as new genomes are added to a taxon, which seems to be a correct approximation [35]. After applying this normalization we found that the most overrepresented taxa in the data are Planctomycetes, Synergistetes, and Firmicutes. Bacteria from these taxa as well as those from Proteobacteria and Bacteroidetes, co-occur in microbial communities with Methanosarcina spp. [37-39].

\section{Functional breakdown of the transferred genes}

To characterize functional consequences of HGT, all identified HT genes were assigned to COG categories, $86 \%$ (506 proteins) of HT genes had COG identifiers. Half of HT genes are associated with metabolism (Table 4). Assuming equal frequency of HGT for all COG categories, the expected transfer rates were based on mean fractions of the COG categories in all bacterial and archaeal genomes. Overrepresented categories include Defense mechanisms (V) and all types of metabolism except lipid metabolism and catabolism: Amino acid transport and metabolism (E), Energy production and conversion (C), Nucleotide transport and metabolism (F), Inorganic ion transport and metabolism (P), Carbohydrate transport and metabolism (G), Secondary metabolites biosynthesis, transport and catabolism (Q), Coenzyme transport and metabolism (H) (Fig. 5a).

Then, HT proteins of Methanosarcina spp. were assigned to four major functional groups: housekeeping genes, enzymes, transporters, and transcriptional regulators. The first category comprises replication, translation and transcription machinery genes, as well as genes encoding cell wall proteins.

The number of transcriptional factors and housekeeping genes in our data was estimated using COGs, while the number of transporters and enzymes was estimated using PFAM and EFICAz databases, respectively.

Seven housekeeping GOPs with HT genes were found: t-RNA-dihydrouridine synthase; cysteinyl-tRNA synthase; two acetyltransferases or methyltransferases, whose function could not be characterized further; and three proteins involved in DNA repair. All other proteins involved in transcription, translation and replication were not found among the HT genes.

Among 226 transcriptional regulators observed in the Methanosarcina spp. only seven, belonging to two GOPs, were identified as HT ones. The fraction of transport proteins in Methanosarcina spp. comprises about $4.4 \%$ of all genes (505 proteins), which is in good agreement with other estimates [40]. More than half of identified HT genes with an assigned functional class were either enzymes or transporters (187 and 67, respectively). The fraction of HT genes among transporters and enzymes was, respectively, $12-15 \%$ and 9-11\% (Table 5). Hence, HGT of transporter genes is slightly more common than the transfer of enzyme genes, although this difference is not statistically significant (Table 5).

These results show that genes involved in metabolism, as well as in defense mechanisms (e.g. restriction-modification system, chloramphenycol O-acetyltransferase and multidrug transporters) are frequently transferred from Bacteria to Archaea. Lipid metabolism and catabolism is the only metabolic subsystem, where HT genes are rare. A natural

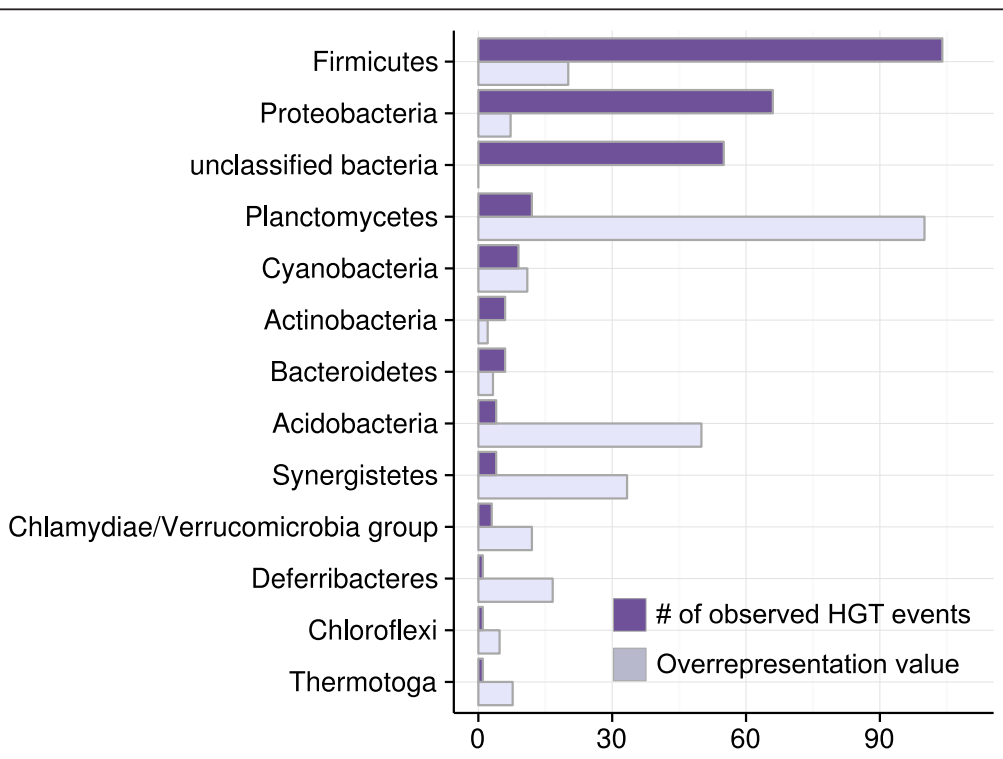

Fig. 4 Taxonomic distribution of transferred genes. Distribution of HGT donors according to number of observed HGT events (purple) and overrepresentation values (lavender); see the text for definitions 
Table 4 Functional distribution of HT genes

\begin{tabular}{|c|c|c|c|c|c|c|c|c|c|c|c|c|c|c|c|}
\hline \multirow{2}{*}{\multicolumn{2}{|c|}{ COG category }} & \multicolumn{6}{|c|}{ Presence in Methanosarcina } & \multicolumn{8}{|c|}{ Presence in Methanosarcinaceae } \\
\hline & & No. & Enz. & Tr. & 1 & 2 & 3 & No & Enz & $\operatorname{Tr}$ & 2 & 3 & 4 & 5 & 6 \\
\hline \multicolumn{2}{|r|}{ Information storage and processing: } & \multicolumn{6}{|c|}{$18(9,7 \%)$} & \multicolumn{8}{|c|}{$7(11,7 \%)$} \\
\hline J & Translation, ribosomal structure and biogenesis & 6 & 3 & 0 & 2 & 2 & 2 & 2 & 1 & 0 & 0 & 0 & 1 & 1 & 0 \\
\hline K & Transcription & 7 & 1 & 0 & 5 & 1 & 1 & 3 & 0 & 0 & 0 & 0 & 0 & 2 & 1 \\
\hline L & Replication, recombination and repair & 5 & 2 & 0 & 2 & 1 & 2 & 2 & 1 & 0 & 0 & 0 & 0 & 1 & 1 \\
\hline \multicolumn{2}{|c|}{ Cellular processes and signaling: } & \multicolumn{6}{|c|}{$26(14 \%)$} & \multicolumn{8}{|c|}{$9(15 \%)$} \\
\hline V & Defense mechanisms & 11 & 7 & 3 & 4 & 5 & 2 & 1 & 1 & 0 & 0 & 1 & 0 & 0 & 0 \\
\hline $\mathrm{T}$ & Signal transduction mechanisms & 5 & 2 & 0 & 3 & 2 & 0 & 1 & 0 & 0 & 0 & 0 & 0 & 0 & 1 \\
\hline M & Cell wall/membrane/envelope biogenesis & 8 & 1 & 0 & 4 & 1 & 3 & 5 & 2 & 2 & 0 & 1 & 2 & 2 & 0 \\
\hline $\mathrm{N}$ & Cell motility & 1 & 1 & 0 & 0 & 1 & 0 & 0 & 0 & 0 & 0 & 0 & 0 & 0 & 0 \\
\hline $\mathrm{O}$ & Posttranslational modification, protein turnover, chaperones & 1 & 0 & 0 & 0 & 1 & 0 & 2 & 0 & 0 & 0 & 0 & 1 & 1 & 0 \\
\hline \multicolumn{2}{|c|}{ Metabolism: } & \multicolumn{6}{|c|}{$95(51,3 \%)$} & \multicolumn{8}{|c|}{$37(54 \%)$} \\
\hline C & Energy production and conversion & 21 & 10 & 0 & 3 & 14 & 4 & 10 & 2 & 0 & 0 & 1 & 6 & 3 & 0 \\
\hline E & Amino acid transport and metabolism & 25 & 10 & 8 & 1 & 13 & 11 & 9 & 5 & 1 & 0 & 0 & 2 & 1 & 6 \\
\hline $\mathrm{F}$ & Nucleotide transport and metabolism & 7 & 5 & 0 & 1 & 4 & 2 & 3 & 2 & 0 & 0 & 0 & 0 & 0 & 3 \\
\hline G & Carbohydrate transport and metabolism & 10 & 2 & 2 & 1 & 4 & 5 & 3 & 1 & 0 & 0 & 1 & 1 & 0 & 1 \\
\hline $\mathrm{H}$ & Coenzyme transport and metabolism & 10 & 3 & 0 & 1 & 7 & 2 & 4 & 1 & 0 & 1 & 0 & 1 & 0 & 2 \\
\hline | & Lipid transport and metabolism & 3 & 0 & 0 & 0 & 1 & 2 & 0 & 0 & 0 & 0 & 0 & 0 & 0 & 0 \\
\hline P & Inorganic ion transport and metabolism & 16 & 2 & 9 & 2 & 9 & 5 & 6 & 0 & 5 & 0 & 0 & 0 & 1 & 5 \\
\hline Q & Secondary metabolites biosynthesis, transport and catabolism & 3 & 0 & 0 & 0 & 2 & 1 & 2 & 1 & 0 & 0 & 0 & 1 & 0 & 1 \\
\hline \multicolumn{2}{|c|}{ Poorly characterized: } & \multicolumn{6}{|c|}{$46(24,8 \%)$} & \multicolumn{8}{|c|}{$16(23,5 \%)$} \\
\hline $\mathrm{R}$ & General function prediction only & 31 & 10 & 2 & 10 & 13 & 8 & 11 & 2 & 0 & 0 & 0 & 3 & 2 & 6 \\
\hline S & Function unknown & 15 & 0 & 0 & 1 & 4 & 10 & 5 & 0 & 0 & 0 & 0 & 1 & 3 & 1 \\
\hline
\end{tabular}

Tr. is the number of GOPs containing transporters, Enz. is the number of GOPs containing enzymes, numbers in the heading represent the number of genomes in GOP. All HT singletons were also included into this analysis

explanation for that is the difference in the membrane composition of Archaea and Bacteria [41]. Overrepresentation of enzymes and transporters among HT proteins confirms that these types of genes are the most common subjects of HGT [9, 13, 35] (Fig. 5b). While HGT of both enzymes [14] and transporters [27] had been described in Archaea, the rates of their transfer were not compared directly.

\section{Operon structure}

To characterize operons (for definition see Materials and Methods), all intergenic distances were calculated for each Methanosarcina genome. Initially six different intergenic spacers' thresholds were analyzed (300, 250, 200, 150, 100 and $50 \mathrm{bp}$ ). A conservative value of $150 \mathrm{bp}$ was selected as the maximum distance between genes in an operon, because with longer intergenic spacers, short HT operons start to merge with non-HT genes, while with a more stringent threshold on spacer length more than half of Methanosarcina genes are not included in operons.

About $67 \%$ of $M$. acetivorans and M. mazei genes, and $65 \%$ of $M$. barkeri genes comprise non-trivial operons.
Of all operons with HT genes (Table 6), 10 operons are common for all Methanosarcina spp. For HT genes, the fraction of genes in operons is slightly lower, significant at $p=0.005$ level (Table 6 and Fig. 5c). The average size of an operon with HT genes is 3.5 genes per operon, while the average operon length in the genome is slightly larger, comprising 4.1 gene per operon. $50 \mathrm{M}$. acetivorans operons, 32 in M. barkeri, and 33 in M. mazei were formed by both HT and archael genes. The mean length of a HT fragment is about 1300 nucleotides (see Materials and Methods), while the longest transferred segment consists of more than 7000 nucleotides, and contains 9 HT Na-ATPase genes. They form one operon, common for $M$. acetivorans and $M$. barkeri, and previously shown to be transferred as a single HGT event [42].

\section{HT genes expression}

Available microarray and proteomics data were analyzed for three Methanosarcina species (see Methods). In four experiments on M. mazei [43-46], 82 of 151 predicted HT genes were shown to be differentially expressed under a variety of conditions (Additional file 1). Thirty 

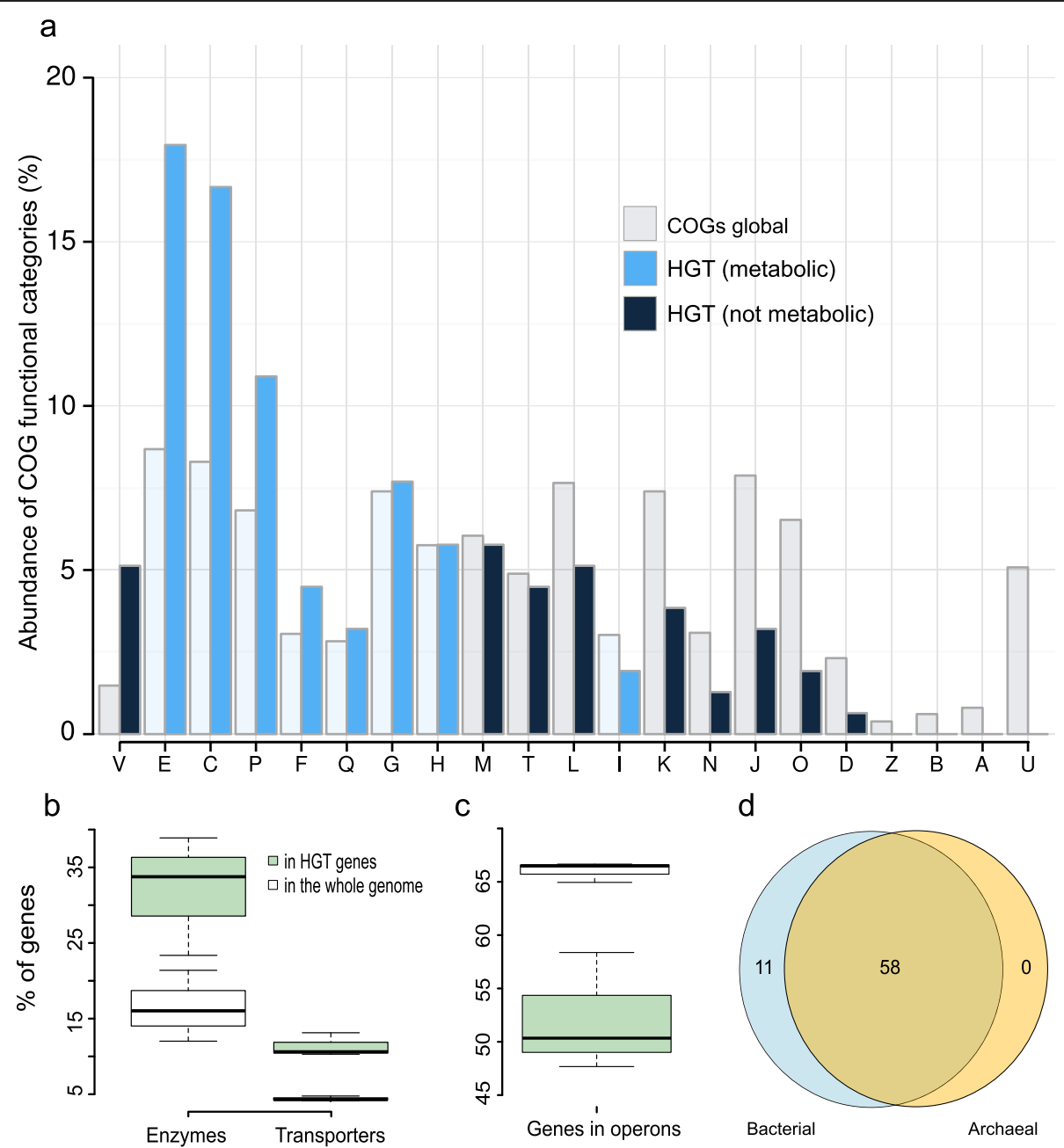

Fig. 5 Functional distribution of transferred genes and their operon structure. a Representation of horizontally transferred genes in COG categories (see the text for definitions). $\mathbf{b}$ The fraction of genes encoding enzymes or transporters among the HT genes and the whole genomes. c The fraction of genes included in operons among the HT genes, and the whole genomes. $\mathbf{d}$ Venn diagram representing the distribution of COGs with transcriptional factors among Archaea and Bacteria

Table 5 Distribution of transporters and enzymes among HT genes and the genome in general

\begin{tabular}{|c|c|c|c|c|c|c|c|}
\hline & \multicolumn{3}{|l|}{ Enzymes } & \multicolumn{3}{|l|}{ Transporters } & \multirow{2}{*}{$\begin{array}{l}x^{2} \text {-test } \\
p \text {-value }\end{array}$} \\
\hline & $\begin{array}{l}\text { \# of proteins in } \\
\text { HT genes }\end{array}$ & $\begin{array}{l}\text { \# of proteins in the } \\
\text { genome }\end{array}$ & $\% \mathrm{HT}$ & $\begin{array}{l}\text { \# of proteins in } \\
\text { HT genes }\end{array}$ & $\begin{array}{l}\text { \# of proteins in the } \\
\text { genome }\end{array}$ & $\% \mathrm{HT}$ & \\
\hline $\begin{array}{l}\text { M. } \\
\text { acetivorans }\end{array}$ & 86 (38.9 \%) & $971(21.4 \%)$ & 8.9 & 29 (13.1\%) & $194(4.3 \%)$ & 14.9 & 0.0094 \\
\hline M. barkeri & $50(23.4 \%)$ & 435 (12\%) & 11.5 & $22(10.3 \%)$ & $172(4.7 \%)$ & 12.8 & 0.6562 \\
\hline M. mazei & $51(33.8 \%)$ & 540 (16 \%) & 9.4 & 16 (10.6\%) & 139 (4.1\%) & 11.5 & 0.4663 \\
\hline All & 187 (31.9\%) & 1946 (16.9 \%) & 9.6 & 67 (11.4\%) & 505 (4.4\%) & 13.3 & 0.0163 \\
\hline
\end{tabular}

Percent value in the brackets represents the \% of enzymes or transporters in studied portion of genome (HT genes or all genes), while \% HT column shows the percentage of occurrence of the proteins from this functional class among HT portion of genome. P-value is calculated for the null hypothesis that fractions of enzymes and transporters are equal among $\mathrm{HT}$ genes and for all genes 
Table 6 Summary of the operon structure in Methanosarcina spp. among HT genes and for the genome in general

\begin{tabular}{|c|c|c|c|c|c|c|c|c|c|}
\hline & \multicolumn{4}{|c|}{ Genome in general } & \multicolumn{4}{|l|}{ HT genes } & \multirow{2}{*}{$\begin{array}{l}x \text { 2-test } \\
p \text {-value }\end{array}$} \\
\hline & Single genes & Genes in operons & \# of operons & $\%$ & Single genes & Genes in HT operons & \# of operons & $\%$ & \\
\hline M. acetivorans & 1543 & 3063 & 768 & $66,5 \%$ & 92 & 129 & 80 & $58,4 \%$ & 0.0087 \\
\hline M. barkeri & 1296 & 2402 & 570 & $65,0 \%$ & 112 & 102 & 61 & $47,7 \%$ & $5 \mathrm{E}-008$ \\
\hline M. mazei & 1145 & 2290 & 543 & $66,7 \%$ & 75 & 76 & 56 & $50,3 \%$ & $1 \mathrm{E}-005$ \\
\hline
\end{tabular}

HT operons are non-trivial operons with at least one HT gene. P-value is calculated for the null hypothesis that the fractions of operons and singletons are the same for HT genes and all genes

of these genes were found to change the level of expression in the absence of histone; 66 genes were differentially expressed depending on the nitrogen source; 6 genes showed increased expression on methanol media; 10, increase on acetate media. Overall, in the M. mazei genome, $55 \%$ of genes were shown to be differentially expressed, so the fraction of observed expressed HT genes is not lower than the genome average. In $M$. acetivorans proteomic data, 13 proteins (6.7 \%) encoded by HT genes were found (for comparison, $9.1 \%$ genes from the complete genome were identified) [47, 48]; in microarray data [49], 17 HT genes $(12.6 \%)$ showed differential expression on methanol or acetate as a food source, while the overall number of differentially expressed genes in this experiments reached $27.5 \%$. For M. barkeri, 7 HT genes (3.6 \%) differentially expressed after air-exposure were found, whereas in the whole genome, 40 (1.6\%) such genes were observed [50]. Overall, $46 \%$ of HT GOPs contain at least one member that was found to be differentially expressed. These results show that HT genes are active in Methanosarcina spp., and at least some genes have been shown to be differentially expressed, and hence are likely regulated.

\section{Discussion}

HGT plays an important role in diversity and adaptation of microorganisms. In Bacteria, HGT initially was shown to be responsible for rapid spread of antibiotic resistance on plasmids [51]. Special gene transfer agents (GTAs) were discovered in the purple nonsulfur bacterium Rhodobacter capsulatus [52], and it was shown that in marine bacterial populations the transfer rate of antibiotic resistance genes included in GTAs was high [53]. Rapid gene acquisitions through HGT are thought to have driven adaptation to different ecological niches $[1,8,54]$ and the origin of new bacterial and archaeal species $[12,14]$.

HGT rates for a variety of prokaryotic and eukaryotic [55] species were estimated. In particular, all members of the Methanosarcina genus were claimed to have dynamic genomes with a high HGT rate $[19,24,25]$. Later, HGT in Methanosarcinales was studied [26, 27], while for the family Methanosarcinaceae this phenomenon was not addressed directly, and the functional distribution of HT genes transferred to the last common ancestor of Methanosarcina spp. or Methanosarcinaceae was not investigated. We estimate that about $5 \%$ of Methanosarcina genes are horizontally transferred from bacteria since the last common ancestor of Methanosarcinaceae. Among them, $3.5 \%$ of genes were transferred to the last common ancestor of Methanosarcina spp. or even later in evolution, and $1.5 \%$ of genes are the result of HGT to the last common ancestor of Methanosarcinaceae. Our estimate of the HGT rate in Methanosarcina generally agrees with the one provided by the arCOG analysis, where 321 HGT events were identified. However, in that study, the origin of the HGT was not identified [26].

In order to understand why the fraction of bacterial HT genes in Methanosarcina spp. has been overestimated in initial publications [19, 24], we performed searches through a series of time-scaled databases, and found that this effect was observed because of the database composition in 2001. Further, we re-evaluated the fraction of HT genes in Thermotoga maritima. Again, we could not reproduce the original result of $24 \%$ HT genes [31], and observed a lower rate of 8-11 \% [33]. Still, it looks like at least some HGT events from Archaea to T. maritima had occurred. We observed 28 such events, which is much lower than thought initially [31, 33, 34, 56]. In both cases, artifacts in previous estimates of the rate of gene flow between Archaea and Bacteria were likely due to incomplete and biased composition of available databases and application of simple BLAST-based procedures that were not sufficiently reliable given these biases. However, it is possible that more HGT events occurred earlier in the Thermotoga or methanogenic archaea evolution, and for Methanosarcinales a high rate of HGT from bacteria was shown [27].

Many HT genes common for $M$. acetivorans and $M$. barkeri are not present in M. mazei. On the phylogenetic tree constructed using ribosomal proteins, $M$. acetivorans and M. mazei form a cluster to the exclusion of M. barkeri. It means that $M$. mazei, whose genome is the smallest one among these three species, has lost many HT genes. While the last common ancestor of Methanosarcina spp. is thought to be a halophile as most Methanosarcinaceae, it could be speculated, that M. mazei gene loss could be associated with adaptation to low salt concentrations, one such example is the loss of $\mathrm{Na}+-$ ATPase and some metal transporters [42].

HGT played a major role in the development of acetoclastic methanogenesis. Examples of such transfer are 
genes coding proteins Pta and AckA, key proteins is acetoclastic methanogenesis, that were thought earlier, when no other Methanosarcinaceae genomes were sequenced except for Methanosarcina spp., were thought to be transferred to the last common of Methanosarcinaceae from Clostridia [14]. We found that only the clade with Methanosarcina proteins is situated within the Clostridia clade, while no orthologs of Pta and AckA have been found in other Methanosarcinaceae (Fig. 1a). This event had likely involved only the last common ancestor of Methanosarcina spp., while other Methanosarcinales use other methanogenesis enzymes.

In theory, HGT events can occur between any prokaryotic groups with similar codon usage [17, 18], but additional factors have to be considered, such as co-occurrence of organisms in the same ecological niches [57], or toxicity of HT gene products. The arCOG study did not reveal any 'highways' of HGT, that would preferentially connect particular groups of archaea and bacteria [58], but we have demonstrated that, at least for Methanosarcina spp., some trends may exist, and HGT from Clostridia and Proteobacteria to Methanosarcina spp. is the most frequent.

As Clostridia and Proteobacteria are the best sequenced groups of Bacteria, we also applied weighted measures to find the most frequent donors outside the best sequenced groups. Clearly, these calculations of overrepresentation are very approximate and have obvious limitations. Indeed, even in groups with hundreds of sequenced genomes, not all available ecological niches are sampled, and such groups often have numerous sequences for popular species. On the other hand, the complicated history of HGT in bacteria may further obscure the real donor. We presented both estimates with and without normalization for sequencing biases, and the truth probably lies somewhere in the middle. Still, while the most frequent donor could not be identified confidently, some robust trends could be observed, such as prevalence of Firmicutes as candidate donors.

We also considered the functional distribution of transferred genes. Both enzymes and transporters are the most frequent subjects of HGT (Fig. 5c). While transporters are more frequently transferred than enzymes, this observation does not reach statistical significance. However, this analysis is blind to archaea-specific transport proteins, that are still not well studied [40]. The addition of such transporters, as well as characterization of new classes of bacterial transporters, may influence the significance of this conclusion. Theoretically, the HGT of transporters is a convenient mechanism of adaptation to rapidly changing conditions, providing the cell with ions and nutrients from a new environment. $\mathrm{ABC}$-transporters are known to be transferred between and within domains of life, and in a situation when such genes have been transferred from bacteria to Methanosarcina via a third, archaeal organism, whose genome is not available, false predictions could be made. Similarly, if a gene has been subject to HGT from Methanosarcina to another archaeal species, our study design would not identify it as transferred from bacteria to Methanosarcina. However, here we concentrated on recent HGT, where such misidentifications are less likely.

While the membrane of Bacteria and Archaea consists of different types of lipids, these results show that bacterial-type transporters could work in both cell types. The only class of transporters underrepresented among HT genes is lipid transporters, similarly to lipid metabolism enzymes, likely reflecting differences in the membrane structures.

The transfer of enzymes is rarer, mainly because a new enzyme has to be incorporated in a pre-existing metabolic pathway, and, further, subunits of protein complexes and proteins with multiple interactions seem to undergo fewer HGT events $[15,16]$.

HGT of bacterial genes has been studied for the last common ancestor of Methanosarcinales (three Methanosarcina genomes, M. burtonii and Methanosaeta thermophila RT) [7], and while the distribution of functional classes is similar with the one that we observe for HGT in the common ancestors of Methanosarcinaceae and Methanosarcina, there are some differences. Firstly, for Methanosarcinales more transfers of genes involved in replication, recombination and reparation (category $\mathrm{L}$ ) have been observed; secondly, there are fewer transporters among transferred genes. The latter may be caused by difficulties in establishing orthology in large gene families, common for transporters, at large evolutionary distances. Also, for relatively recent events we observed that half of the transferred genes are involved in metabolism, while in the Methanosarcinales study [27] only $26 \%$ of gains are associated with metabolism. Hence, the presented results show that relatively recent HGT events tend to impact the metabolic potential, but not the basic cell functions, such as replication or recombination.

More than a half of HGT genes found in M. mazei were shown to be functional and differentially expressed, and the important question is how all these genes are regulated. The total number of known transcription factors in Methanosarcinales corresponds to earlier predictions for prokaryotic organisms [59]. This observation may be biased by the fact that the number of transcription factors was estimated using the COG classification, that contains no clusters comprised exclusively by archaeal TFs, while many TF clusters are bacteria-specific (Fig. 5d). Only in two HGT events TFs were actually transferred, but about $46 \%$ of GOPs were shown to be expressed, and if the regulators tend to be transferred together with the genes they regulate, we would expect to see more HGT 
events involving TFs. This leads to a conclusion that HT genes from bacteria do not tend to be transferred with their regulators, but are mainly regulated by the factors already existing in the acceptor organism.

As we have considered only recent HGT events, we do not expect massive genome rearrangements since HGT, and that has allowed us to analyze the operon structure of HT genes. Among HT genes, we have observed a lower fraction of operons, and the characteristic length of the transferred fragments is about $1200 \mathrm{bp}$ (the average length of one protein-coding gene). Moreover, there are also mosaic operons comprised of both HT and archaeal genes. It is possible that the length of a HT fragment is limited by the mechanism of gene transfer from bacteria to archaea.

\section{Conclusions}

Previously, the role of HGT in Methanosarcina spp. was overestimated due to biased data. A more robust estimate of the fraction of HT genes either in the last common ancestor of Methanosarcina spp. or of Methanosarcinaceae is $\sim 5 \%$. We studied the operon structure of HT genes and showed that the HT genes do not tend to be transferred as whole operons. Most frequent HGT donors are Firmicutes and Proteobacteria. While the regulation of HT genes is not well understood, about half of identified HT genes in $M$. mazei are differentially expressed.

\section{Methods}

\section{Genomes}

Genome sequences were downloaded from Genbank (www.ncbi.nlm.nih.gov).

Four groups of species were used: (1) Methanosarcina spp. (M. acetivorans C2A (NC_003552; NC_002097) [24], M. barkeri str. Fusaro (NC_007349) [25], M. mazei Go1 (NC_007355; NC_003901) [19]); (2) all Methanosarcinaceae available as of March, 2012 (Methanosarcina spp., Methanococcoides burtonii DSM 6242 (NC_007955) [20], Methanohalobium evestigatum Z-7303 (NC_014253), Methanohalophilus mahii DSM 5219 (NC_014002) [21]); (3) Pyrococcus spp. (P. horikoshii OT3 (NC_000961) [60], P. abyssi GE5 (NC_000868; NC_001773) [61], P. furiosus DSM 3638 (NC_003413) [62]); and (4) all available Thermotogaceae (Thermotoga thermarum DSM 5069 (NC_015707), Thermotoga sp. RQ2 (NC_010483.1) [63], Thermotoga petrophila RKU-1 (NC_009486.1) [33], Thermotoga neapolitana DSM 4359 (NC_011978.1), Thermotoga naphthophila RKU-10 (NC_013642), Thermotoga maritima MSB8 (NC_000853) [31], Thermotoga lettingae TMO (NC_009828), Thermosipho melanesiensis BI429 (NC_009616), Thermosipho africanus TCF52B (NC_011653), Petrotoga mobilis SJ95 (NC_010003), Kosmotoga olearia TBF 19.5.1 (NC_012785) [64], Fervidobacterium nodosum Rt17-B1 (NC_009718)).

\section{Grouping of orthologs}

Initially, groups of orthologous proteins (GOPs) were constructed for every pair of species in a group. All pairwise comparisons were done using BLASTP [65], and bidirectional best hits (BBHs) were identified. Hits were ignored if the identity level was less than $50 \%$ (40\% for Methanosarcinaceae) or if the aligned region was less than $2 / 3$ of the length of the shorter protein. Then, if two paralogous genes from one genome were more similar to each other than to a BBH partner from another genome, both were added to the orthology group. Then, maximal connected components were constructed. The groups were formed using ad-hoc software written using Oracle RDBMS Express Edition (PL/SQL codes are available in Additional file 2).

\section{Identification of HGT}

To identify HGT events, several perl scripts were developed. Each member of a GOP was used as a query in a BLAST search against the non-redundant protein sequences database (release 2011-07-16) with default parameters. Organisms were classified according to the NCBI taxonomy [66].

If all proteins of an analyzed GOP had three top hits only in the Bacteria superkingdom or only three among twenty top hits were archaeal, while all others were bacterial (with identity cut-off at least $30 \%$, length of HSP not less than 50, and coverage of a Methanosarcinaceae protein by a bacterial hit not less than $75 \%$ ), the GOP was retained for further analysis.

At the next step, top 100 protein hits for each member of the GOP were selected. The selected proteins for all GOP members were aligned by MUSCLE (version 3.6) [67] with default settings. The alignment quality was controlled by GUIDANCE [68] and manually. If more than half of columns in an alignment had score less than default GUIDANCE cutoff (0.93), such GOPs were excluded from the analysis. For each alignment, two types of trees were constructed, a neighbor-joining (NJ) tree with 100 bootstrap replicas using the ClustalW software with default parameters (BLOSUM distance matrix) [69], and a maximum-likelihood (ML) tree using PhyML v3.0 with default parameters [70]. Trees were visualized using the iTOL server [71].

$\mathrm{NJ}$ and ML trees were analyzed independently. No special rooting procedures were applied, but if nonMethanosarcinaceae archaea formed a monophyletic clade on the tree, the root was placed manually between these archaea and bacteria. If no such monophyletic clade was present or only Methanosarcinaceae archaea were present, the root was placed in the point of divergence of large bacterial taxa. If for both $\mathrm{NJ}$ and ML trees all studied Methanosarcinaceae genes formed a stable subclade (for NJ tree with bootstrap $>70$ ) within a bacterial clade, 
without other archaeal proteins, such GOPs were considered as candidate HGT. For each HGT event, organisms closest to Methanosarcinaceae on the tree were analyzed to identify the source and timing of the HGT event. It should be noted, however, that both individual decisions in each particular case and overall conclusions are robust as regards the exact position of the root, unless it is positioned on a branch between the Methanosarcinaceae clade and the rest of the tree.

A HGT event was considered to be a transfer to the last common ancestor of Methanosarcina spp., if all members of the Methanosarcina GOP, consisting of at least two Methanosarcina spp. proteins from different species, formed a stable clade only with bacterial proteins, and no other archaeal proteins were present on that clade, but they could be present elsewhere in the tree. HGT to Methanosarcinaceae was detected if all members of the Methanosarcinaceae GOPs (at least two proteins of Methanosarcina species, and least one protein from other Methanosarcinaceae species) are grouped with bacteria and not with other archaea. The source of HGT was identified as the lowest common taxonomic rank for all bacteria that form a stable clade with GOP members.

To analyze orphan genes not included in any GOPs and find recent HGT events in the Methanosarcina species the same procedure was applied.

The BLAST-based pipeline include only BLAST search, as described above, without further tree construction.

\section{Phylogenetic trees of Methanosarcinaceae}

Concatenated alignment of ribosomal proteins S2, S5, L2, L3, L4, L6, two copies of S4, and protein EF-TuA as in [72] was generated using the MUSCLE software. The rRNA alignments were downloaded from the SILVA database [73]. These alignments were used to produce neighbor-joining trees with 100 bootstrap replicas by ClustalW software, as described above.

\section{Assigning proteins to COG}

Each predicted HGT protein was assigned to an orthologous group in the COG IMG database [74]. For each studied organism, the overrepresentation $(\mathrm{O})$ for a COG category was measured as:

$$
O=\frac{C O G_{o b s}-C O G_{\text {exp }}}{C O G_{\exp }}
$$

where $C O G_{\text {exp }}$ is the normalized number of COGs in the COG category among all archael or bacterial genes, and $C O G_{o b s}$ is the number of observed COGs in this category among the HT genes.

\section{Assigning proteins to functional classes}

The fraction of transcription regulators was estimated separately for each Methanosarcina species by calculating the number of proteins in the COG category "Transcription regulation".

The fraction of transport proteins in Methanosarcina proteomes was estimated using PFAM [75, 76]. The total number of proteins assigned to PFAM families was calculated for each complete Methanosarcina proteome and also for those proteins that were clustered into GOPs. All Methanosarcina proteins were extracted from PFAM families with keyword 'transport' in the family name, and the fractions of transport proteins were calculated for proteins clustered into all GOPs, and for proteins clustered in HT GOPs. The fraction of enzymes in the complete proteome, in GOPs, and in HT GOPs was estimated by the EFICAz EC classification [77]. All Methanosarcina entries with EC annotation were extracted from the database and then enzyme functions were assigned to the HT genes and all proteins in the Methanosarcina GOPs using NCBI gi identifiers.

\section{Operon prediction (selection of parameters, comparison with microarray data)}

All gene coordinates and directions were extracted from Genbank genome annotations. Intergenic distances were calculated using perl scripts. Operons were defined as sets of adjacent codirectional genes with short intergenic spacers, with the thresholds discussed in the Results section. The length of HT fragment was estimated by a sum of lengths of sequential HT genes and intergenic spacers between them. For trivial operons, the length of HT fragment equals to gene length. Singleton HT genes were excluded from this analysis.

To analyze the expression of HT genes, all available experimental microarray and proteomics data were analyzed [43-50]. Genes were considered to be expressed, if significant expression was observed in microarray experiments, or corresponding proteins were found in proteome analyses.

\section{Dependence on the database composition}

To evaluate the annual change of the predicted HGT rate in the Methanosarcina spp., all archaeal completely sequenced genomes were extracted from Genbank, and the year of sequencing was recorded for each genome. A series of year-stamped databases based on BLASTP nonredundant database were made from year 2001 through 2011, in which all archaea that were sequenced after the given year were masked. BLASTP searches for each GOP member were made with default parameters against yearstamped databases. 


\section{Availability of supporting data}

The data sets supporting the results of this article are available online in the Dryad data repository under doi: http://dx.doi.org/10.5061/dryad.j69p2 [78], and other datasets supporting the results of this article are provided with Additional files.

\section{Additional files}

Additional file 1: List of HT genes found in Methanosarcina spp. Additional file 2: ZIP archive with PL/SQL scripts used to assemble groups of orthologous proteins.

\section{Competing interests}

The authors declare that they have no competing interests.

\section{Authors' contributions}

MSG conceived and coordinated the project. MDK constructed orthologous groups. SKG developed the procedure to predict horizontal gene transfer, and analyzed the results. MSG, SKG and MDK wrote the manuscript. All authors read and approved the final manuscript.

\section{Acknowledgements}

We are grateful to Dmitry Vinogradov and Olga Tsoy for useful discussions. This study was supported by the Russian Science Foundation (grant 14-24-00155).

Received: 28 March 2014 Accepted: 29 May 2015

Published online: 05 June 2015

\section{References}

1. Ochman H, Lawrence JG, Groisman EA. Lateral gene transfer and the nature of bacterial innovation. Nature. 2000;405:299-304.

2. Omelchenko MV, Makarova KS, Wolf YI, Rogozin IB, Koonin EV. Evolution of mosaic operons by horizontal gene transfer and gene displacement in situ. Genome Biol. 2003;4:R55.

3. Wozniak RAF, Waldor MK. Integrative and conjugative elements: mosaic mobile genetic elements enabling dynamic lateral gene flow. Nat Rev Microbiol. 2010;8:552-63.

4. Babic A, Berkmen MB, Lee CA, Grossman AD. Efficient gene transfer in bacterial cell chains. mBio. 2011;2:e00027-11-e00027-11.

5. Polz MF, Alm EJ, Hanage WP. Horizontal gene transfer and the evolution of bacterial and archaeal population structure. Trends Genet. 2013:29:170-5.

6. Popa O, Dagan T. Trends and barriers to lateral gene transfer in prokaryotes. Curr Opin Microbiol. 2011;14:615-23. Antimicrobials/Genomics.

7. Sjostrand J, Tofigh A, Daubin V, Arvestad L, Sennblad B, Lagergren J. A bayesian method for analyzing lateral gene transfer. Syst Biol. 2014;63:409-20.

8. Gogarten JP, Townsend JP. Horizontal gene transfer, genome innovation and evolution. Nat Rev Microbiol. 2005;3:679-87.

9. Choi I-G, Kim S-H. Global extent of horizontal gene transfer. Proc Natl Acad Sci. 2007;104:4489-94.

10. Koonin EV. Horizontal gene transfer: the path to maturity. Mol Microbiol. 2003;50:725-7.

11. Novichkov PS, Omelchenko MV, Gelfand MS, Mironov AA, Wolf YI, Koonin EV. Genome-wide molecular clock and horizontal gene transfer in bacterial evolution. J Bacteriol. 2004;186:6575-85.

12. Nelson-Sathi $S$, Sousa FL, Roettger M, Lozada-Chávez $N$, Thiergart $T$, Janssen A, Bryant D, Landan G, Schönheit P, Siebers B, Mclnerney JO, Martin WF. Origins of major archaeal clades correspond to gene acquisitions from bacteria. Nature. 2015;517(7532):77-80.

13. Creevey CJ, Doerks T, Fitzpatrick DA, Raes J, Bork P. Universally distributed single-copy genes indicate a constant rate of horizontal transfer. PLoS One. 2011;6, e22099.

14. Fournier GP, Gogarten JP. Evolution of acetoclastic methanogenesis in Methanosarcina via horizontal gene transfer from cellulolytic clostridia. J Bacteriol. 2007;190:1124-7.

15. Jain R, Rivera MC, Lake JA. Horizontal gene transfer among genomes: the complexity hypothesis. Proc Natl Acad Sci. 1999;96:3801-6.
16. Wellner A, Lurie MN, Gophna U. Complexity, connectivity, and duplicability as barriers to lateral gene transfer. Genome Biol. 2007;8:R156.

17. Medrano-Soto A. Successful lateral transfer requires codon usage compatibility between foreign genes and recipient genomes. Mol Biol Evol. 2004;21:1884-94.

18. Sorek R, Zhu Y, Creevey CJ, Francino MP, Bork P, Rubin EM. Genome-wide experimental determination of barriers to horizontal gene transfer. Science. 2007;318:1449-52.

19. Deppenmeier U, Johann A, Hartsch T, Merkl R, Schmitz RA, Martinez-Arias R, et al. The genome of Methanosarcina mazei: evidence for lateral gene transfer between bacteria and archaea. J Mol Microbiol Biotechnol. 2002;4:453-61.

20. Allen MA, Lauro FM, Williams TJ, Burg D, Siddiqui KS, De Francisci D, et al. The genome sequence of the psychrophilic archaeon, Methanococcoides burtonii: the role of genome evolution in cold adaptation. ISME J. 2009:3:1012-35.

21. Spring S, Scheuner C, Lapidus A, Lucas S, Glavina Del Rio T, Tice H, et al. The genome sequence of Methanohalophilus mahii SLPT reveals differences in the energy metabolism among members of the Methanosarcinaceae inhabiting freshwater and saline environments. Archaea. 2010;2010:1-16.

22. Anderson I, Ulrich LE, Lupa B, Susanti D, Porat I, Hooper SD, et al. Genomic characterization of Methanomicrobiales reveals three classes of methanogens. PLoS One. 2009;4, e5797.

23. Fournier GP, Huang J, Gogarten JP. Horizontal gene transfer from extinct and extant lineages: biological innovation and the coral of life. Philos Trans R Soc B Biol Sci. 2009;364:2229-39.

24. Galagan JE. The genome of $M$. Acetivorans reveals extensive metabolic and physiological diversity. Genome Res. 2002;12:532-42.

25. Maeder DL, Anderson I, Brettin TS, Bruce DC, Gilna P, Han CS, et al. The Methanosarcina barkeri genome: comparative analysis with Methanosarcina acetivorans and Methanosarcina mazei reveals extensive rearrangement within methanosarcinal genomes. J Bacteriol. 2006;188:7922-31.

26. Wolf Yl, Makarova KS, Yutin N, Koonin EV. Updated clusters of orthologous genes for Archaea: a complex ancestor of the Archaea and the byways of horizontal gene transfer. Biol Direct. 2012;7:1-15.

27. Nelson-Sathi S, Dagan T, Landan G, Janssen A, Steel M, Mclnerney JO, et al. Acquisition of 1,000 eubacterial genes physiologically transformed a methanogen at the origin of Haloarchaea. Proc Natl Acad Sci. 2012;109:20537-42.

28. Simankova MV, Parshina SN, Tourova TP, Kolganova TV, Zehnder AJ, Nozhevnikova AN. Methanosarcina lacustris sp. nov., a new psychrotolerant methanogenic archaeon from anoxic lake sediments. Syst Appl Microbiol. 2001;24:362-7

29. Rouvière $P$, Mandelco L, Winker $S$, Woese CR. A detailed phylogeny for the Methanomicrobiales. Syst Appl Microbiol. 1992;15:363-71.

30. Gao B, Gupta RS. Phylogenomic analysis of proteins that are distinctive of Archaea and its main subgroups and the origin of methanogenesis. BMC Genomics. 2007:8:86

31. Nelson KE, Clayton RA, Gill SR, Gwinn ML, Dodson RJ, Haft DH, et al. Evidence for lateral gene transfer between Archaea and bacteria from genome sequence of Thermotoga maritima. Nature. 1999:399:323-9.

32. Logsdon JM, Faguy DM. Evolutionary genomics:Thermotoga heats up lateral gene transfer. Curr Biol. 1999:9:R747-51.

33. Zhaxybayeva O, Swithers KS, Lapierre P, Fournier GP, Bickhart DM, DeBoy RT, et al. On the chimeric nature, thermophilic origin, and phylogenetic placement of the Thermotogales. Proc Natl Acad Sci. 2009;106:5865-70.

34. Nesbo CL, L'Haridon S, Stetter KO, Doolittle WF. Phylogenetic analyses of two "archaeal" genes in Thermotoga maritima reveal multiple transfers between archaea and bacteria. Mol Biol Evol. 2001;18:362-75.

35. Koonin EV, Wolf YI. Genomics of bacteria and archaea: the emerging dynamic view of the prokaryotic world. Nucleic Acids Res. 2008;36:6688-719.

36. Gordienko EN, Kazanov MD, Gelfand MS. Evolution of Pan-Genomes of Escherichia coli, Shigella spp., and Salmonella enterica. J Bacteriol. 2013;195:2786-92.

37. Wang $H$, Vuorela $M$, Keränen $A-L$, Lehtinen TM, Lensu $A$, Lehtomäki $A$, et al. Development of microbial populations in the anaerobic hydrolysis of grass silage for methane production. FEMS Microbiol Ecol. 2010;72:496-506.

38. Tang Y-Q, Fujimura Y, Shigematsu T, Morimura S, Kida K. Anaerobic treatment performance and microbial population of thermophilic upflow anaerobic filter reactor treating awamori distillery wastewater. J Biosci Bioeng. 2007;104:281-7.

39. Patil SS, Kumar MS, Ball AS. Microbial community dynamics in anaerobic bioreactors and algal tanks treating piggery wastewater. Appl Microbiol Biotechnol. 2010;87:353-63. 
40. Ren Q, Paulsen IT. Comparative analyses of fundamental differences in membrane transport capabilities in prokaryotes and eukaryotes. PLoS Comput Biol. 2005;1, e27.

41. De Rosa M, Gambacorta A, Gliozzi A. Structure, biosynthesis, and physicochemical properties of archaebacterial lipids. Microbiol Rev. 1986:50:70-80.

42. Dibrova DV, Galperin MY, Mulkidjanian AY. Characterization of the N-ATPase, a distinct, laterally transferred $\mathrm{Na}+$-translocating form of the bacterial F-type membrane ATPase. Bioinformatics. 2010;26:1473-6.

43. Hovey R, Lentes S, Ehrenreich A, Salmon K, Saba K, Gottschalk G, et al. DNA microarray analysis of Methanosarcina mazei Gö1 reveals adaptation to different methanogenic substrates. Mol Genet Genomics. 2005;273:225-39.

44. Veit K, Ehlers C, Ehrenreich A, Salmon K, Hovey R, Gunsalus RP, et al. Global transcriptional analysis of Methanosarcina mazei strain Gö1 under different nitrogen availabilities. Mol Genet Genomics. 2006;276:41-55.

45. Pfluger K, Ehrenreich A, Salmon K, Gunsalus RP, Deppenmeier U, Gottschalk $G$, et al. Identification of genes involved in salt adaptation in the archaeon Methanosarcina mazei GÃ 1 using genome-wide gene expression profiling FEMS Microbiol Lett. 2007:277:79-89.

46. Weidenbach K, Glöer J, Ehlers C, Sandman K, Reeve JN, Schmitz RA. Deletion of the archaeal histone in Methanosarcina mazei Gö1 results in reduced growth and genomic transcription. Mol Microbiol. 2008;67:662-71.

47. Li Q, Li L, Rejtar T, Karger BL, Ferry JG. Proteome of Methanosarcina a cetivorans part I: an expanded view of the biology of the cell. J Proteome Res. 2005;4:112-28.

48. Li Q, Li L, Rejtar T, Karger BL, Ferry JG. Proteome of Methanosarcina a cetivorans part II: comparison of protein levels in acetate- and methanol-grown cells. J Proteome Res. 2005:4:129-35.

49. Li L, Li Q, Rohlin L, Kim U, Salmon K, Rejtar T, et al. Quantitative proteomic and microarray analysis of the archaeon Methanosarcina acetivorans grown with acetate versus methanol. J Proteome Res. 2007;6:759-71.

50. Zhang W, Culley DE, Nie L, Brockman FJ. DNA microarray analysis of anaerobic Methanosarcina barkeri reveals responses to heat shock and air exposure. J Ind Microbiol Biotechnol. 2006;33:784-90.

51. Datta N, Kontomichalou P. Penicillinase synthesis controlled by infectious $R$ factors in Enterobacteriaceae. Nature. 1965;208:239-41.

52. Lang AS, Beatty JT. Importance of widespread gene transfer agent genes in a-proteobacteria. Trends Microbiol. 2007:15:54-62.

53. McDaniel LD, Young E, Delaney J, Ruhnau F, Ritchie KB, Paul JH. High frequency of horizontal gene transfer in the oceans. Science. 2010;330:50.

54. Cohan FM, Koeppel AF. The origins of ecological diversity in prokaryotes. Curr Biol CB. 2008;18:R1024-1034

55. Crisp A, Boschetti C, Perry M, Tunnacliffe A, Micklem G. Expression of multiple horizontally acquired genes is a hallmark of both vertebrate and invertebrate genomes. Genome Biol. 2015;16.

56. Mongodin EF, Hance IR, DeBoy RT, Gill SR, Daugherty S, Huber R, et al. Gene transfer and genome plasticity in Thermotoga maritima, a model hyperthermophilic species. J Bacteriol. 2005;187:4935-44.

57. Schliep K, Lopez P, Lapointe F-J, Bapteste E. Harvesting evolutionary signals in a forest of prokaryotic gene trees. Mol Biol Evol. 2010;28:1393-405.

58. Puigbò $P$, Wolf $Y$ I, Koonin EV. Seeing the tree of life behind the phylogenetic forest. BMC Biol. 2013;11:46.

59. Maslov S, Krishna S, Pang TY, Sneppen K. Toolbox model of evolution of prokaryotic metabolic networks and their regulation. Proc Natl Acad Sci. 2009;106:9743-8.

60. Kawarabayasi Y, Sawada M, Horikawa H, Haikawa Y, Hino Y, Yamamoto S, et al. Complete sequence and gene organization of the genome of a hyper-thermophilic archaebacterium, Pyrococcus horikoshii OT3. DNA Res Int J Rapid Publ Rep Genes Genomes. 1998;5:55-76.

61. Cohen GN, Barbe V, Flament D, Galperin M, Heilig R, Lecompte O, et al. An integrated analysis of the genome of the hyperthermophilic archaeon Pyrococcus abyssi. Mol Microbiol. 2003;47:1495-512.

62. Robb FT, Maeder DL, Brown JR, DiRuggiero J, Stump MD, Yeh RK, et al. Genomic sequence of hyperthermophile, Pyrococcus furiosus: implications for physiology and enzymology. Methods Enzymol. 2001;330:134-57.

63. Swithers KS, DiPippo JL, Bruce DC, Detter C, Tapia R, Han S, et al. Genome sequence of Thermotoga sp. strain RQ2, a hyperthermophilic bacterium isolated from a geothermally heated region of the seafloor near Ribeira Quente, the Azores. J Bacteriol. 2011;193:5869-70.

64. Swithers KS, DiPippo JL, Bruce DC, Detter C, Tapia R, Han S, et al. Genome sequence of Kosmotoga olearia strain TBF 19.5.1, a thermophilic bacterium with a wide growth temperature range, isolated from the Troll B oil platform in the North Sea. J Bacteriol. 2011;193:5566-7.

65. Altschul SF, Madden TL, Schäffer AA, Zhang J, Zhang Z, Miller W, et al. Gapped BLAST and PSI-BLAST: a new generation of protein database search programs. Nucleic Acids Res. 1997;25:3389-402.

66. NCBI taxonomy [http://www.ncbi.nlm.nih.gov/taxonomy]

67. Edgar RC. MUSCLE: multiple sequence alignment with high accuracy and high throughput. Nucleic Acids Res. 2004;32:1792-7.

68. Penn O, Privman E, Ashkenazy H, Landan G, Graur D, Pupko T. GUIDANCE: a web server for assessing alignment confidence scores. Nucleic Acids Res. 2010;38(Web Server):W23-8.

69. Larkin MA, Blackshields G, Brown NP, Chenna R, McGettigan PA, McWilliam H, et al. Clustal W and Clustal X version 2.0. Bioinforma Oxf Engl. 2007;23:2947-8.

70. Guindon S, Dufayard J-F, Lefort V, Anisimova M, Hordijk W, Gascuel O. New algorithms and methods to estimate maximum-likelihood phylogenies: assessing the performance of PhyML 3.0. Syst Biol. 2010;59:307-21.

71. Letunic I, Bork P. Interactive tree of life v2: online annotation and display of phylogenetic trees made easy. Nucleic Acids Res. 2011;39 suppl 2:W475-8.

72. McCutcheon JP, Moran NA. Extreme genome reduction in symbiotic bacteria. Nat. Rev. Microbiol. 2012;10:13-26.

73. Quast C, Pruesse E, Yilmaz P, Gerken J, Schweer T, Yarza P, et al. The SILVA ribosomal RNA gene database project: improved data processing and web-based tools. Nucleic Acids Res. 2012;41:D590-6.

74. COG IMG database [https://img.jgi.doe.gov/cgi-bin/w/main.cgi?section=Find Functions\&page=ffoAllCogCategories]

75. Finn RD, Bateman A, Clements J, Coggill P, Eberhardt RY, Eddy SR, et al. Pfam: the protein families database. Nucleic Acids Res. 2014;42:D222-30.

76. Pfam database [http://pfam.xfam.org/]

77. Arakaki AK, Huang Y, Skolnick J. EFICAz2: enzyme function inference by a combined approach enhanced by machine learning. BMC Bioinformatics. 2009;10:107.

78. Garushyants SK, Kazanov MD, Gelfand MS. Data from: horizontal gene transfer and genome evolution in Methanosarcina. Dryad Digital Repository. doi:10.5061/dryad.j69p2.

\section{Submit your next manuscript to BioMed Central and take full advantage of:}

- Convenient online submission

- Thorough peer review

- No space constraints or color figure charges

- Immediate publication on acceptance

- Inclusion in PubMed, CAS, Scopus and Google Scholar

- Research which is freely available for redistribution

Submit your manuscript at www.biomedcentral.com/submit 